\title{
The relationship between wear and dissipated energy in sliding systems
}

\author{
A. Ramalho ${ }^{\text {a,* }}$, J.C. Miranda ${ }^{b}$ \\ ${ }^{a}$ Universidade de Coimbra-ICEMS-Dep. Eng. Mecânica, Pólo II, Pinhal de Marrocos, P-3030-201 Coimbra, Portugal \\ ${ }^{\mathrm{b}}$ Esc. Sup. de Tecnologia e Gestão, Inst. Politec. da Guarda, 6300 Guarda, Portugal \\ Received 15 April 2004; accepted 3 February 2005 \\ Available online 10 May 2005
}

\begin{abstract}
Wear is the progressive loss of material from the operating surface of a body occurring as a result of relative motion at the surface, while friction is the main means of controlling the input of energy in a system. The tribosystem approach proposes analysing the contact on both the material and the energetic planes, but the relationships between the different planes are not well established. In this paper, the basis of the energetic approach is discussed and the application of the model to experimental studies on bulk materials reveals a promising and powerful tool to analyse experimental results and to use in the mechanical design.
\end{abstract}

(C) 2005 Elsevier B.V. All rights reserved.

Keywords: Wear; Friction; Dissipated energy

\section{Introduction}

Wear is the progressive loss of material from the operating surface of a body, caused by relative motion at the surface. Wear may be broadly classified as mild or severe. In mild wear, the process occurs at the outer surface layers, the surfaces remain smooth and are usually covered by surface oxides generated during rubbing. Mild wear results in small track debris, down to some nanometers. In severe wear, the contact is metallic, the surfaces are seriously deformed and the worn debris consists of particles up to some hundred micrometers. Therefore, this simplest classification is primarily a distinction based in the scale of size.

The more traditional classification of wear is based on the relative type of motion or in the type of wear mechanism [1]. Under the former classification, wear can be classified as wear by sliding, rolling, impact, oscillation and flow. Concerning wear mechanisms, the more usual are: adhesion, abrasion, surface fatigue and tribochemical.

The wear in a tribological contact often involves a complex interdependence between several wear mechanisms. The

\footnotetext{
* Corresponding author. Tel.: +351 239790756; fax: +351 239790701 .

E-mail address: amilcar.ramalho@dem.uc.pt (A. Ramalho).
}

research work set out to discuss the basis of a new approach to the wear of materials for experimental studies and to apply it as a design tool.

\section{Theoretical concepts}

\subsection{Development of the model}

The wear in a tribological contact involves a complex interdependence between several processes, including chemical and mechanical interactions, and depends on many physical parameters. As it is very difficult to isolate an individual parameter or process, the system approach can be a very important technique for analysing tribomechanical contacts.

Czichos [2] gives a clear description of the principles underlying the application of system concepts to the analysis of tribological contacts. At a qualitative level, at least, concepts similar to thermodynamic analysis could be applied to contact problems. A system may generally be defined as a set of elements interconnected by structure and function. Therefore, given a suitable structure ( $A$ elements with $P$ relevant properties and $R$ relations between them), the system works as an operator suitable for transforming a set of inputs $\{X\}$ 
into outputs $\{Y\}$. The dynamic behaviour of tribosystems can be characterized by means of a generalized balance of energy. This means that the net energy of the system remains constant if we consider the process of storing and transforming energy.

Tribosystems analysis could be carried out using a threedimensional approach, taking [2]:

- the functional aspect, considering the system as an operator which transforms the set of inputs $\{X\}$ in the outputs $\{Y\}$;

- the energy aspect, analysing the exchanges and transformations between thermal and mechanical energy, as well as the entropy variation;

- the material aspect, studying the exchange of materials between the two first bodies and the interfacial volume.

The changes in each of the dimensions (or planes) analysed can affect the balance in the others. For example, mechanical energy can be affected by temperature and can influence the behaviour in the material plane. The complex tribology of mechanical systems results in both the interdisciplinary nature of the problem, and the complexity of the relationships between the different planes (functional, energetic and material). In most of the systems, inputs and outputs include the flow of materials through the system. The wear results from losses of the elements from the system, which can lead to property changes in the system itself. Considering that friction is the most important process related to changes in the system's energy, it inevitably plays an important role in the losses of the system's elements.

Classical theories give the wear volume $(V)$ proportional to the normal load $(N)$, the sliding distance $(S)$ and the inverse of the hardness $(H)$, Eq. (1). The wear coefficient $K$ establishes the proportionality and can be used as a measure of the material wear behaviour [3]. Both $K$ and $H$ reveal the material response and thus can be grouped in a single parameter, called the specific wear rate, $k=V / S N$, that can be used instead of the wear coefficient $K$, giving Eq. (2):

$V=K \frac{S N}{H}$

$V=k S N$

The Coulomb friction model, Eq. (3), establishes that the friction force is proportional to the normal applied load, $N$. Therefore, assuming a constant friction coefficient, a proportional relationship, can be established between the wear volume and the friction force, Eq. (4):

$F=\mu N$

$V \propto F S$

For unidirectional sliding, the second term of expression (4) represents the work of the friction force. Therefore, from the energetic approach, the volume of wear appears to be directly proportional to the energy dissipated by friction. This fact demonstrates the existence of a relationship between the wear and the friction.
Therefore, considering the tribosystem approach, the energy dissipated by friction seems to be the relationship between the material and the energetic planes.

The energy dissipated per unit of contact area was first introduced in the 1960s as the friction power intensity concept,

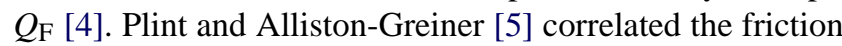
power intensity with the time of the friction action with the derivation of another concept, the energy pulse, EP. The energy pulse only applies in such contacts where the contact point is moved relative to both surfaces, and encouraging values were obtained that could be used to describe contacts like those occurring with cam-followers and gearing teeth [5]. As the EP is not suitable for the contacts often used in tribological characterization, Celis and co-workers $[6,7]$ introduced the concept of dissipated energy for the work done by the friction force during the entire test, and developed procedures to calculate the cumulative energy in fretting tests. The suitability of the dissipated energy for explaining the effect of different environmental parameters has been demonstrated for coated materials [6-9]. Huq and Celis [10] have shown that the same principle of dissipated energy could be applied successfully to unidirectional tests. In fact, for TiN coatings tested against alumina balls with normal loads from 3 to $15.8 \mathrm{~N}$, a linear relationship between the wear volume and the dissipated energy was found [10].

Updating the concept of energy dissipated per unit of contact area recent papers by Fouvry and co-workers [11,12] introduced a new concept: the energy density parameter. The relationship between the energy density parameter and the wear depth was demonstrated for coated surfaces under fretting contacts [12]. The ability of the energy density parameter to predict the wear evolution proves that the energetic approach could be a promising design tool for contacting surfaces.

The friction energy in the contact is dissipated mainly through three processes: rise in temperature; wear particle generation; and the entropy changes associated with material transformation in the interface. By numerical simulation, Fouvry et al. [11] concludes that the particle generation and oxidation were the contact processes that consume more energy. The postulate on which the dissipated energy model is based is that if the wear mechanism remains the same, the input of energy in the tribosystem is divided in such a way that the relative amounts of the different main parts of the energy consumption remain constant. Nowadays, the use of energetic concepts, particularly the use of the $P V$ limit as a design criterion, is well established in tribology. In fact for a wide range of materials, a relationship can be established between $P V$ and the wear rate. Assuming a constant friction coefficient, $P V$ is proportional to the specific power dissipated by friction, with SI units of $\mathrm{W} / \mathrm{m}^{2}$. In reciprocated tests, especially in fretting tests, the energy dissipated by friction also is now widely used to characterize the material response. Therefore, this study uses energetic concepts to develop a systematic procedure that can be used to analyse the results of unidirectional sliding tests. 
The energetic approach is more than a new way to analyse the wear data, it is actually a complete approach to the study of tribological contacts. The major advantages of that approach are:

- its suitability as design tool. In fact, if the friction coefficient is available, the relationship between energy and wear volume being known, the loss of material through wear could be predicted;

- the simplification in wear testing. Using the energetic model, the classical experimental data analysis procedures, where the same test conditions are repeated to calculate an average value, can be replaced by a new approach where different test parameters, including test duration, can be compared because they affect both the input of energy and the wear volume in the same way.

\section{Experimental work}

\subsection{Experimental details}

The energy dissipated by friction was studied experimentally using a sliding tribometer with crossed cylinder contact, Fig. 1. The equipment includes a rotating cylinder (3) and a cylindrical stationary specimen (5). The normal load is applied by means of a screw/spring system (4) and is measured by a load cell (1). The stationary specimen, whose diameter is $10 \mathrm{~mm}$, is supported by a free rotating system, which is equilibrated by a load cell used to measure the friction force (2). The diameter of the rotating disc was $60 \mathrm{~mm}$ and the rotation speed was $159 \mathrm{rpm}$, and the sliding speed was therefore $0.5 \mathrm{~m} / \mathrm{s}$.

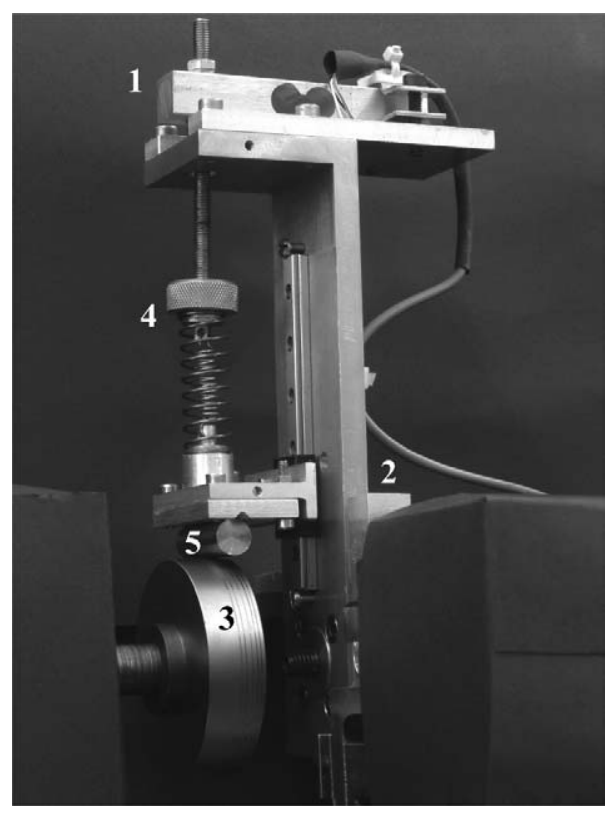

Fig. 1. Sliding tribometer with crossed cylinder contact.
This experimental study was designed to determine whether an energetic approach can be used to characterise the wear resistance of materials under sliding contacts. Three different bulk materials were thus selected, namely: mild steel AISI 1037, hard steel AISI 52100 and tungsten carbide with $10 \mathrm{wt} . \%$ of cobalt. The rotating specimen was always the same, hard high-speed-steel AISI M2.

The normal load ranged from 1 to $6 \mathrm{~N}$ and the test duration was from 20 to $90 \mathrm{~min}$, which corresponds to a sliding distance of $600-2700 \mathrm{~m}$.

Before testing, the specimens were cleaned with ethyl alcohol. During the test, the friction force value was acquired periodically, at time intervals of $\Delta t$. In each acquisition, a set of several thousand of values was collected, corresponding to an acquisition time greater than the rotation period. Therefore, the average value of the friction force, $\bar{F}$, calculated from the acquired friction force data, corresponds to the average of the friction during a rotation.

The energy dissipated in the contact can be calculated as the work of the friction force. For each time interval $\Delta t$, which corresponds to a displacement $\Delta x$, the dissipated energy $\Delta E$ can be found with Eq. (5). Considering the mean value of the friction force and assuming a constant sliding speed, Eq. (6) can be used:

$$
\begin{aligned}
& \Delta E=\int_{0}^{\Delta t} F \mathrm{~d} x=\int_{0}^{\Delta t} F V \mathrm{~d} t \\
& \Delta E=\bar{F} V \Delta t
\end{aligned}
$$

The total energy dissipated throughout the test can be calculated by adding all the $\Delta E$ calculated during the course of the test. At the end of the test, the stationary specimen shows an elliptical wear scar, Fig. 2. In addition, the wear produces a track in the rotating specimen.

For the stationary specimen, the volume of the wear scar can be calculated assuming an imposed wear shape using the approximate expression (7) derived by Ramalho [13]. This

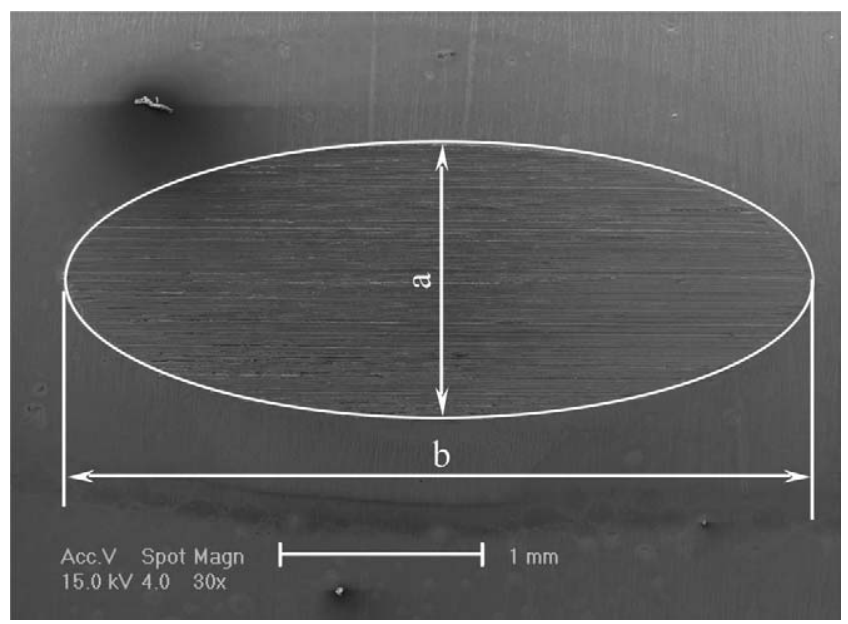

Fig. 2. Typical wear scar for the stationary specimen. 
Table 1

Wear results of all the performed tests

\begin{tabular}{|c|c|c|c|c|c|}
\hline Materials & Case & Normal load (N) & Rotations & Wear volume $\left(\mathrm{m}^{3}\right)$ & Dissipated energy $(\mathrm{J})$ \\
\hline \multirow[t]{5}{*}{ AISI M2-AISI 1037} & 1 & 1 & 4770 & $3.36 \mathrm{E}-11$ & 603.92 \\
\hline & 2 & 2 & 4770 & $5.99 \mathrm{E}-11$ & 1039.09 \\
\hline & 3 & 3 & 3180 & $9.64 \mathrm{E}-11$ & 996.02 \\
\hline & 4 & 4 & 3180 & $1.31 \mathrm{E}-10$ & 1333.76 \\
\hline & 5 & 5 & 3180 & $1.86 \mathrm{E}-10$ & 1800.78 \\
\hline \multirow[t]{5}{*}{ AISI M2-AISI 52100} & 6 & 2 & 3180 & $8.40 \mathrm{E}-12$ & 493.93 \\
\hline & 7 & 2 & 14310 & $1.22 \mathrm{E}-10$ & 3434.11 \\
\hline & 8 & 4 & 3180 & $4.56 \mathrm{E}-11$ & 1309.05 \\
\hline & 9 & 4 & 14310 & $1.83 \mathrm{E}-10$ & 6233.35 \\
\hline & 10 & 6 & 3180 & $7.16 \mathrm{E}-11$ & 2694.57 \\
\hline \multirow[t]{5}{*}{ AISI M2-WC } & 11 & 4 & 14310 & $5.40 \mathrm{E}-12$ & 6537.11 \\
\hline & 12 & 5 & 4770 & $3.90 \mathrm{E}-12$ & 3357.7 \\
\hline & 13 & 5 & 14310 & $7.60 \mathrm{E}-12$ & 11618.82 \\
\hline & 14 & 6 & 4770 & $4.50 \mathrm{E}-12$ & 3030.47 \\
\hline & 15 & 6 & 9540 & $5.40 \mathrm{E}-12$ & 5820.89 \\
\hline
\end{tabular}

very simple equation is very accurate with errors of less than $0.2 \%$ [13]:

$V=\frac{\pi}{2} h^{2} \sqrt{d_{1} d_{2}}$

Where $d_{1}$ is the diameter of the stationary specimen; $d_{2}$ the diameter of the rotating specimen; and $h$ the depth of the scar. Each scar is measured by taking the larger, $a$, and the smaller, $b$, dimensions of the wear surface. The value of scar depth can be calculated either by Eq. (8) or (9). The mean wear depth, $h$, can be evaluated using the average value of $h_{1}$ and $h_{2}$, Eq. (10):

$$
\begin{aligned}
& h_{1}=\frac{d_{1}}{2}\left(1-\cos \left(\arcsin \left(\frac{a}{d_{1}}\right)\right)\right) \\
& h_{2}=\frac{d_{2}}{2}\left(1-\cos \left(\arcsin \left(\frac{b}{d_{2}}\right)\right)\right) \\
& h=\frac{h_{1}+h_{2}}{2}
\end{aligned}
$$

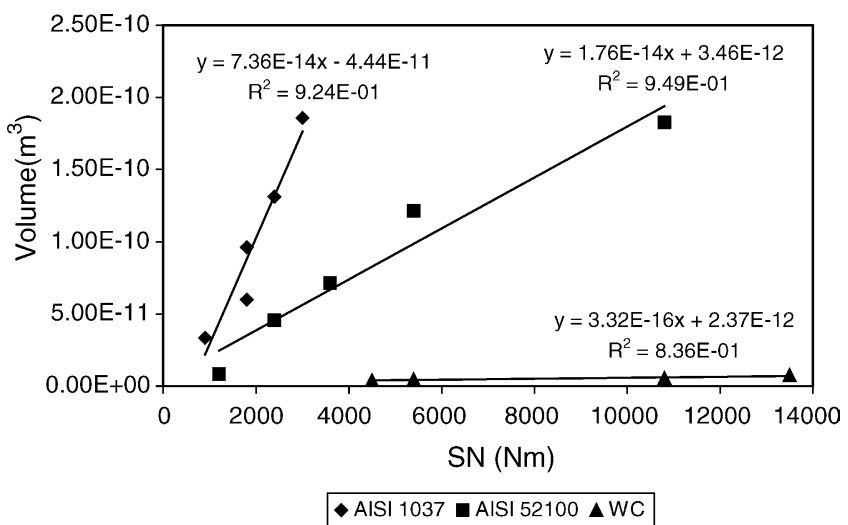

Fig. 3. Wear volume against $S N$ for the three tested materials.

\subsection{Results and discussion}

The wear of the moving specimens was very low, whatever the test conditions, so accurate measurement was not possible. There were two reasons for this: highly wear-resistant material was selected for the moving specimen; and the wear was not point-concentrated as for the stationary specimen, it

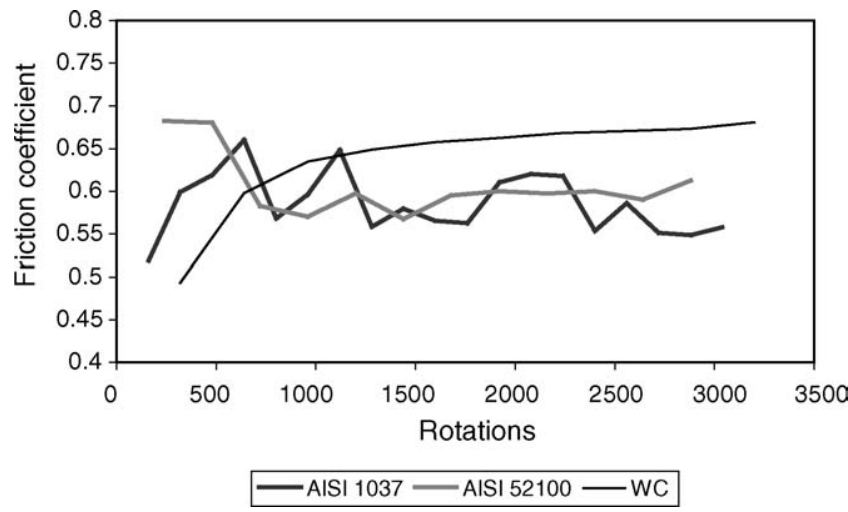

Fig. 4. Evolution of the friction coefficient during the test.

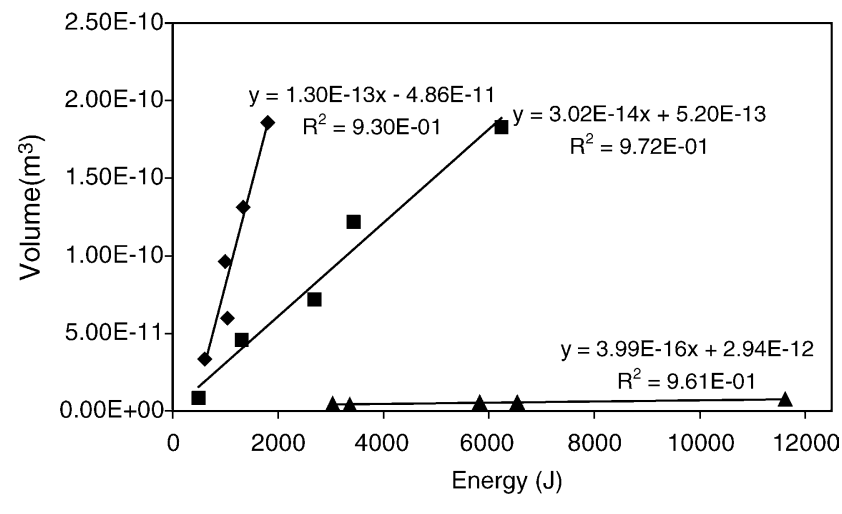

$\bullet$ AISI 1037 aISI $52100 \Delta$ WC

Fig. 5. Wear volume against dissipated energy for the three tested materials. 

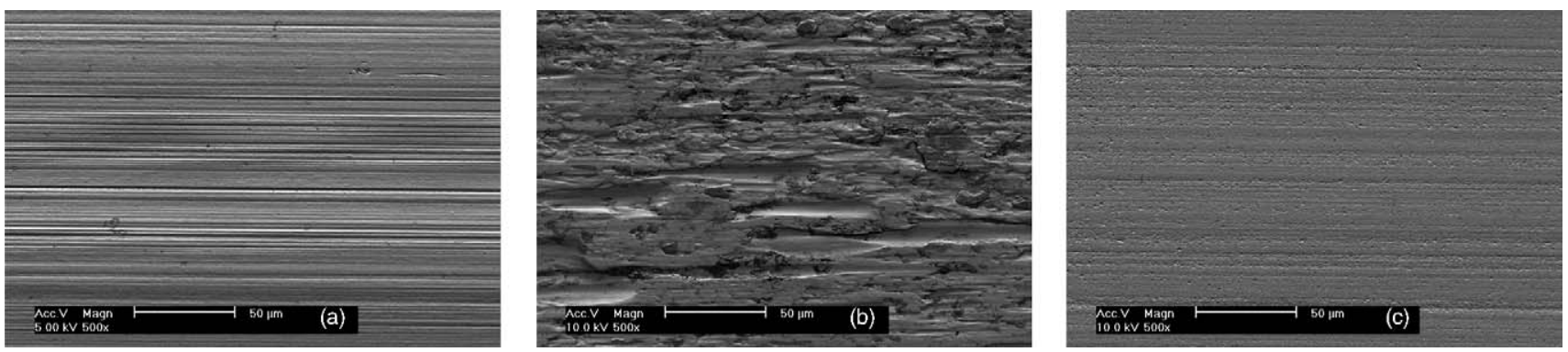

Fig. 6. Morphology of wear surfaces: (a) AISI 52100 steel; (b) AISI 1037 steel; (c) tungsten carbide.

was spread throughout the external perimeter of the moving specimen. Therefore, only the wear of the stationary specimen has been calculated, using Eq. (7). The dissipated energy has been calculated for each test, using Eq. (6) and the procedure described above. Table 1 shows the results for all the tests.

In order to evaluate the ability of the energetic approach, the results were first analysed by means of the classical approach. Thus, based on the Archard equation, the specific wear rate can be calculated as $k=V / S N$, where $S$ and $N$ are respectively the sliding distance and the normal load. Fig. 3 shows the results obtained using that approach. The specific wear rate varies from $1.30 \times 10^{-13} \mathrm{~m}^{2} / \mathrm{N}$ for the mild steel, to $3.99 \times 10^{-16} \mathrm{~m}^{2} / \mathrm{N}$ for tungsten carbide.

The evolution of the friction coefficient was analysed for tests where conditions were the same for all the tested pairs, that is, normal load of $4 \mathrm{~N}$ and test duration of 3180 rotations, Fig. 4. The tungsten carbide shows the highest friction value. While the AISI 1037 steel shows a very irregular friction coefficient all through the test; the other two tested materials tend to a steady state regime after about the first 1000 rotations.

The procedure described previously, based on the dissipated energy, was applied to all the tested cases. The results presented in Fig. 5 reveal linear relationships between the volumes removed by wear and the dissipated energy, with $k_{\mathrm{E}}$ being the specific wear-rate in terms of dissipated energy. The existence of a linear relationship between the volume and the dissipated energy makes a new approach to the wear design of sliding surfaces possible. In fact, if there is an accurate value for the friction coefficient, and if the contact conditions are properly established, the energy dissipated can be calculated and, finally, the wear volume can be achieved using the $k_{\mathrm{E}}$ value. Comparison of Figs. 3 and 5 shows that the correlation of the linear fit is even better for the energetic approach than for the classical approach. Table 2 presents the specific wear rates for both approaches, i.e. function of the $S N$ value and function of the energy. The difference between the biggest values, obtained for the 1037 steel, and the lowest, for the WC in both cases, is of three orders of magnitude in energetic approach and only two orders in the classic approach. In terms of units, the two approaches are similar and have the same units $\left(\mathrm{m}^{2} / \mathrm{N}\right)$, while an average value of $k_{\mathrm{E}}$ can be
Table 2

Specific wear rates as a function of (sliding distance $\times$ normal load) or dissipated energy

\begin{tabular}{lll}
\hline & $\begin{array}{l}\text { Classic approach, } k, \\
\left(\mathrm{~m}^{2} / \mathrm{N}\right)\end{array}$ & $\begin{array}{l}\text { Energy approach, } k_{\mathrm{E}}, \\
\left(\mathrm{m}^{2} / \mathrm{N}\right)\end{array}$ \\
\hline AISI 1037 & $7.36 \times 10^{-14}$ & $1.30 \times 10^{-13}$ \\
AISI 52100 & $1.76 \times 10^{-14}$ & $3.02 \times 10^{-14}$ \\
WC + Co & $3.32 \times 10^{-16}$ & $3.99 \times 10^{-16}$ \\
\hline
\end{tabular}

achieved from $k$, by the quotient of $k$ by the average value of the friction coefficient.

Despite the big difference of the wear coefficients of the several tested materials, the morphology of the wear surfaces reveals that the wear occurs in all cases by abrasion, Fig. 6 . However, some differences were observed: the AISI 52100 steel reveals only a plastic deformation mechanism with fine scratches parallel to the sliding direction, Fig. 6a; the AISI 1037 steel displays a severe grooving superposed with some oxidation, Fig. 6b; finally, the tungsten carbide shows a mild abrasion with some grain detachment, Fig. 6c.

One important advantage of the energetic approach is that it can be applied to thin-coated surfaces. In fact, at least in theory, the model can always be applied, regardless of the test duration, and so tests of different duration can be compared, even when a steady state regime has not yet been reached. Actually the energetic approach takes into account the evolution of the friction coefficient; therefore present higher stability than the Archard formulation. Thus, the energetic approach offer special advantages to study contacts with unstable friction.

\section{Conclusions}

In this paper, the basis of the energetic approach is discussed in order to support its use as a general method to characterize the tribological material response.

The energetic approach is a powerful tool for analysing the data of experimental studies.

One important advantage of the energetic approach is that it can be applied to thin-coated surfaces to compare the results of tests of different duration, even if a steady state regime has not yet been reached. 
The energy approach is also a very useful tool for mechanical design. Although it shares the same basis as the $P V$ factor, the energetic model is more powerful because a prediction of working life can be made directly.

\section{Acknowledgement}

This work was carried out under a project funded by the Portuguese Foundation for Science and Technology (POCTI/33710/EME/2000).

\section{Appendix A. Influence of measurement errors on the resulting values of wear}

The effect of the uncertainty of the measurements of the wear scars and of the specimens on the wear volume has been analysed by applying the general theory of errors propagation [14] to Eqs. (8-10). In the notation used, the same letters used for each value, preceded by delta, represents the errors of each term:

$$
\begin{aligned}
& \Delta h_{1}=\left|\frac{\partial h_{1}}{\partial d_{1}}\right|_{M} \Delta d_{1}+\left|\frac{\partial h_{1}}{\partial a}\right|_{M} \Delta a, \\
& \Delta h_{2}=\left|\frac{\partial h_{2}}{\partial d_{2}}\right|_{M} \Delta d_{1}+\left|\frac{\partial h_{2}}{\partial b}\right|_{M} \Delta b, \\
& \Delta h=\left|\frac{\partial h}{\partial h_{1}}\right|_{M} \Delta h_{1}+\left|\frac{\partial h}{\partial h_{2}}\right|_{M} \Delta h_{2}, \\
& \Delta V=\left|\frac{\partial V}{\partial h}\right|_{M} \Delta h+\left|\frac{\partial V}{\partial d_{1}}\right|_{M} \Delta d_{1}+\left|\frac{\partial V}{\partial d_{2}}\right|_{M} \Delta d_{2}
\end{aligned}
$$

where

$$
\begin{aligned}
& \frac{\partial h_{1}}{\partial d_{1}}=\frac{1}{2}\left[1-\cos \left(\arcsin \left(\frac{a}{d_{1}}\right)\right)\right.-\sin \left(\arcsin \left(\frac{a}{d_{1}}\right)\right) \sqrt{\left.\frac{a^{2}}{d_{1}^{2}-a^{2}}\right]} \\
& \frac{\partial h_{2}}{\partial d_{2}}=\frac{1}{2}\left[1-\cos \left(\arcsin \left(\frac{b}{d_{2}}\right)\right)\right. \\
&\left.\quad-\sin \left(\arcsin \left(\frac{b}{d_{2}}\right)\right) \sqrt{\frac{b^{2}}{d_{2}^{2}-b^{2}}}\right] \\
& \frac{\partial h_{1}}{\partial a}=\frac{d_{1}}{2} \sin \left(\arcsin \left(\frac{a}{d_{1}}\right)\right) \frac{1}{\sqrt{d_{1}^{2}-a^{2}}} \\
& \frac{\partial h_{2}}{\partial b}=\frac{d_{2}}{2} \sin \left(\arcsin \left(\frac{b}{d_{2}}\right)\right) \frac{1}{\sqrt{d_{2}^{2}-b^{2}}} \\
& \frac{\partial h}{\partial h_{1}}=\frac{\partial h}{\partial h_{2}}=\frac{1}{2}
\end{aligned}
$$

Table 3

Influence of the measurement errors on the wear volume

\begin{tabular}{llllll}
\hline & \multicolumn{2}{l}{ Case 5} & & \multicolumn{2}{l}{ Case 12} \\
\cline { 2 - 3 } \cline { 6 - 6 } & Error (\%) & Error & & Error $(\%)$ & Error \\
\hline$\Delta h_{1}$ & 0.26 & $1.82 \mathrm{E}-04$ & & 0.90 & $6.22 \mathrm{E}-05$ \\
$\Delta h_{2}$ & 0.71 & $4.93 \mathrm{E}-04$ & & 1.50 & $1.56 \mathrm{E}-04$ \\
$\Delta h$ & 0.48 & $3.37 \mathrm{E}-04$ & & 1.13 & $1.09 \mathrm{E}-04$ \\
$\Delta V$ & 1.03 & $1.91 \mathrm{E}-03$ & & 2.32 & $9.04 \mathrm{E}-05$ \\
\hline
\end{tabular}

$\frac{\partial V}{\partial h}=\pi h \sqrt{d_{1} d_{2}}$

$\frac{\partial V}{\partial d_{1}}=\frac{\pi h^{2} d_{2}}{4 \sqrt{d_{1} d_{2}}}$

$\frac{\partial V}{\partial d_{2}}=\frac{\pi h^{2} d_{1}}{4 \sqrt{d_{1} d_{2}}}$

Taking into account the procedures used to measure the specimens and the scar, the measurement errors are

$\Delta d_{1}, \Delta d_{2}=10 \mu \mathrm{m} \quad \Delta \mathrm{a}, \Delta \mathrm{b}=5 \mu \mathrm{m}$

Applying the error analysis to the results obtained in the present study, the maximum and the minimum errors correspond to the cases 5 and 12 , respectively (Table 3 ).

The errors in the volume range from 1.03 to $2.32 \%$, and so, taking into account the error of the approximate Equation (8), the main source of the uncertainty is the measurement and not the approximation.

\section{References}

[1] J.T. Burwell, Survey of possible wear mechanisms, Wear 1 (1957) 119-141.

[2] H. Czichos, Tribology-a systems approach to science and technology of friction, lubrication and wear Tribology Series, vol. 1, Elsevier, 1978.

[3] J.F. Archard, Contact and rubbing of flat surfaces, J. Appl. Phys. 24 (1953) 981-988.

[4] R.M. Matveesky, The critical temperature of oil with point and line contact machines, Trans. ASME 87 (1965) 754.

[5] M.A. Plint, A.F. Alliston-Greiner, The energy pulse: a new criterion and its relevance to wear in gear teeth and automotive engine valve trains, Lubr. Sci. 8 (3) (1996) 233-251.

[6] H. Mohrbacher, B. Blanpain, J.-P. Celis, J.R. Roos, The influence of humidity on the fretting behaviour of PVD TiN coatings, Wear 180 (1995) 43-52.

[7] H. Mohrbacher, B. Blanpain, J.-P. Celis, J.R. Roos, L. Stals, M. Van Stappen, Oxidational wear of TiN coatings on tool steel and nitrided tool steel in unlubricated fretting, Wear 188 (1995) 130-137.

[8] S. Fouvry, P. Kapsa, H. Zahouani, L. Vincent, Wear analysis in fretting of hard coatings through a dissipated energy concept, Wear 203/204 (1997) 393-403.

[9] A. Ramalho, J.-P. Celis, High temperature fretting behavior of PVD TiN coatings, Surf. Coat. Technol. 155 (2002) 169-175.

[10] M.Z. Huq, J.-P. Celis, Expressing wear rate in sliding contacts based on dissipated energy, Wear 252 (2002) 375-383. 
[11] S. Fouvry, T. Liskiewicz, P. Kapsa, S. Hannel, E. Sauger, An energy description of wear mechanisms and its application to oscillating sliding contact, Wear 255 (2003) 287-298.

[12] T. Liskiewicz, S. Fouvry, Development of a friction energy capacity approach to predict the surface coating endurance under complex oscillating sliding conditions, Tribol. Int. 38 (2005) 69-79.
[13] A. Ramalho, Micro-scale abrasive wear test of thin coated cylindrical substrates, Tribol. Lett. 16 (1) (2004) 133-141.

[14] J.L. Buchanan, R. Peter, Turner, in: Numerical Methods and Analysis, McGraw-Hill International Editions, 1992. 